\title{
Correction to: Accumulation of potentially harmful elements (PHEs) in lettuce (Lactuca sativa L.) and coriander (Coriandrum sativum L.) irrigated with wastewater: a systematic review and meta-analysis and probabilistic health risk assessment
}

\author{
Ali Atamaleki ${ }^{1} \cdot$ Ahmadreza Yazdanbakhsh $^{2,3} \cdot$ Sevda Fallah $^{3} \cdot$ Mohsen Hesami Arani $^{4,5} \cdot$ Aliasghar Neshat $^{6}$. \\ Yadolah Fakhri ${ }^{7}$ \\ Published online: 1 March 2021 \\ (C) Springer-Verlag GmbH Germany, part of Springer Nature 2021
}

Correction to: Environmental Science and Pollution Research https://doi.org/10.1007/s11356-020-12105-z

The complete name of the 4th Author is presented in this paper.

Publisher's note Springer Nature remains neutral with regard to jurisdictional claims in published maps and institutional affiliations.

The online version of the original article can be found at https://doi.org/ $10.1007 / \mathrm{s} 11356-020-12105-\mathrm{Z}$

\section{Ahmadreza Yazdanbakhsh Yazdanbakhsh@sbmu.ac.ir \\ $\triangle$ Yadolah Fakhri \\ Ya.Fakhri@hums.ac.ir}

1 Student Research Committee, School of Public Health and Safety, Shahid Beheshti University of Medical Sciences, Tehran, Iran

2 Workplace Health Promotion Research Center, Shahid Beheshti University of Medical Sciences, Tehran, Iran

3 School of Public Health and Safety, Shahid Beheshti University of Medical Sciences, Tehran, Iran

4 Student Research Committee, Iran University of Medical Sciences, Tehran, Iran

5 Department of Environmental Health Engineering, School of Public Health, Iran University of Medical Sciences, Tehran, Iran

6 Esfarayen Faculty of Medical Science, Esfarayen, Iran

7 Food Health Research Center, Hormozgan University of Medical Sciences, Bandar Abbas, Iran 